\title{
Presumably Common Trigger Mechanism of Action of Diametrically Different Carcinogens on Target Cells
}

\author{
G.K.Gogichadze", T.G.Gogichadze, G.K.Kamkamidze \\ Department of Microbiology and Immunology, Tbilisi State Medical University, 33, Vazha-Pshavela Ave., Tbilisi, 01776, Georgia \\ *Corresponding Author: gogi_gogichadze@yahoo.com
}

Copyright (C) 2013 Horizon Research Publishing All rights reserved.

\begin{abstract}
A large number of carcinogens and the wide spectrum of their actions may indicate their ability to trigger a common, general mechanism inside the normal biological program of somatic cells, which can lead to the development of the carcinogenic transformation of the normal cell. Numerous carcinogenic substances and factors of different natures probably can signify that these carcinogens must start up some common mechanism of normal cells transition into transformed states. Influence of physical, chemical, and biological carcinogenic agents on cells probably are adequate. By our opinion the common mechanism of action of diametrically different carcinogens on target-cells is the destruction of the plasmatic membrane. After influence of different carcinogens, cells' fusion originates as a result of cytoplasmic membranes perforations (or modifications), i.e. formation in plasma membranes pores, what induces alteration of summary superficial charge of cells' surface. Because of these, cells acquire ability to approach each other (adhesion), what in many cases may be a premise for fusion process. Thus, the initial target of carcinogenic agents is cells' plasma membrane, but not the genetic apparatus of cells.
\end{abstract}

Keywords Carcinogens, Mechanisms of Carcinogenesis, Plasmatic Membrane, Adhesion

\section{Introduction}

Conversion of normal cells into tumorous ones can be induced by the very different in their nature factors. Among them are numerous factors of physical (penetrating radiation of different nature), biological (toxins of different kind, infectious and oncogenic viruses and so on) and chemical nature (there are known more than 1500 carcinogenic chemical substances and combinations; their number constantly increases), what is confirmed by the data of experimental and clinical oncology and epidemiological analysis [1]. The same effect is induced by some pharmacological agents, oral contraceptives, irritations, burns, traumas, chronic inflammations and so on. A large number of carcinogens and the wide spectrum of their actions may indicate their ability to trigger a common, general mechanism inside the normal biological program of somatic cells, which can lead to the development of the neoplastic transformation of the normal cell. Numerous carcinogenic substances and factors of different natures enumerated above probably can signify that these carcinogens must start up some common mechanism of normal cells transition into transformed states.

What are the common signs of all these above-mentioned factors, so different by their nature, as, for example, chemical substances, viruses, radiation of different kinds, glucose, plastics? Is the mechanism of the cells' malignant transformation adequate to the influence of the very different carcinogenic and noncarcinogenic factors?

\section{Hypothesis}

By our opinion the common mechanism of action of diametrically different carcinogens on target-cells is the destruction of the plasmatic membrane (induction of the different size perforations) and consequently development of fusogeny - fusion of somatic cells, which is characteristics for any somatic cells and finally development of karyogamy. Among the processes in which the normal eukaryotic somatic cell-built programs participate during the body's vital activity, the following should be mentioned: mitosis, differentiation, interphase, phagocytosis, endomitosis, apoptosis, necrobiosis, adhesion, and, certainly fusing. When speaking about the biological essence of fusing, its possibility to create polyploidy in somatic cells (like endomitosis) that intensifies resistance of a respective organ to negative environmental factors deserves mentioning. Regrettably, this necessary on the face of it for the organism process is associated with the risks of malignant transformation of the fusion-developed binuclear and polyploidy cells.

In the opinion of some scientists, especially of adherents of virus-genetic theory of carcinogenesis, it is hard to imagine that influence of cellular surface by different 
carcinogens may alter cells' genome; moreover, that inherited character of malignant transformation supposed exactly the cellular genome alteration [2]. From the position of the karyogamic theory, the action of any carcinogen is not associated directly with gene apparatus of cells. Alteration of the cell's genome is induced indirectly; that is, after the somatic cells' fusion and arising of precancerous, tetraploid cell.

As it is known, somatic cells never interact. There is always some type of space (intercellular space) between them which is roughly 100-200 A. As a result each cell maintains its autonomy as an independent unit. As it is thought the balance between the attraction and repulsion should be maintained at this 100-200 A interval. If for any reason the interval become less than $10 \mathrm{~A}$, formation of $\mathrm{Ca}$ bridges is starting leading to the long-term adhesion of the cell. Intercellular contacts are predominantly determined by two main factors: Van der Vaals (positive taxis) and electrostatic (negative taxis) forces contributing to the formation of membrane electric potential. It seems that pores in the plasma membrane on the somatic cells formed by the action of the different physical, chemical and biological agents, substantially decrease the negative charge of the plasma membrane. In different terms, this process (perforation) leads to the weakening on the electrostatic forces and enhancement of the Van der Vaals forces helping somatic cells to overcome intercellular forces and enter into contact with each other. In the case of prolonged contact adhesion process will start to develop [3].

Thus, Influence of physical, chemical, and biological carcinogenic agents on cells probably are adequate. After their influence, cells' fusion originates as a result of cytoplasmic membranes perforations (or modifications), i.e. formation in plasma membranes pores, what induces alteration of summary superficial charge of cells' surface. Because of these, cells acquire ability to approach each other (adhesion), what in many cases may be a premise for fusion process. Thus, the initial target of carcinogenic agents is cells' plasma membrane, but not the genetic apparatus of cells.

Carcinogens' doses probably have the secondary importance in the origin of tumorous cells; moreover, the high doses of carcinogens of different natures (as we see above) can become the reason of the destruction and followinf elimination of the somatic cells and precancerous, initiated cells, as well.

In some cases, carcinogenic agents induce twice or even triple cytopathogenic action on somatic cells: oncogenic (by formation of dikaryons), but in some cases, they induce arising of polykaryocytes and cytolysis in somatic cells (for instance, in immunocompetent cells), which in some cases induce immune deficite of different degrees [4-7]. It is possible, that such different effects of carcinogens on cells depend on size of plasma membranes' pores. In the case of big-sized pores' irreversible changes and cytolysis take place. For instance, high electrostimulation and prolonged impulses lead to partial increasing of quantity of polynuclear cells, but further increase of stimulation induces cellular lysis. In low dose of electrostimulation dikaryons are observed most frequently, but polykaryocytes are revealed, too. Thus, practically all the agents and influences favoring cell's plasma membranes perforations, are regarded as the possible causes of the formation of malignant tumors.

Thus, at the first stage of carcinogenesis, i.e. at the stage of initiation, two (or more) normal somatic cells of one organ or tissue (so-called target-organ or tissue for some kind of the carcinogenic agents) at some favourable conditions may create dikaryons (hetero- or homokaryons), but in some cases nonviable polykaryocytes, i.e. multinuclear cells as well. Presumably, during the perforation of cellular membranes induced by different carcinogenic and noncarcinogenic factors, the total charge of plasma membranes changes, and the cells acquire the capability of closely approaching (adhesion), which frequenty, especially upon coincidence of the perforated parts, may serve as a prerequisite to fusion. At this stage, together with multinuclear cellular structures, the binuclear - hetero- or homokaryons - carriers of high carcinogenic potency are formed. As a result of karyogamy, i.e. after synchronous mitosis or simple mechanical assembly of nuclei heterokaryons (or homokaryons) mononuclear hybrid precancerous cells developes, with tetraploid (or hypotetraploid) set of chromosomes on initial stage of hybridization. Received as a result of somatic hybridization, the hybrid synkaryon is an initiated, immortal, precancerous cell, which exists in macro organism indefinitely for a long time. At the initiated stage of its formation, tumor synkaryon evidently possess tetraploid or hypotetraploid set of chromosomes. Fusion immediately doubles the number of chromosomes, thereby decreasing the chances that the loss of some chromosomes will kill the hybrid cell (synkaryon of stage I). Further, in the processes of promotion and tumor progression, after the segregation of some chromosomes, there may arise tumorous cells with aneuploid or even hyperdiploid set of chromosomes. In extremely rare cases, tumorous cells possess even diploid, hypodiploid or even hyperhaploid sets of chromosomes.

On the promotion stage, after the influence of complete (full) carcinogens or promoters on tissue, where precancerous synkaryons preexist, in these cells the chromosomal aberrations of different types and genes amplifications may arise. One of the conditions in formation of both precancerous and tumorous cells are quantitative (tetraploid, subtetraploid, hyperdiploid, aneuploid and other types of sets of chromosomes) aberrations of chromosomes. The important attending process of cells' malignant conversion on promotion stage is hyperplasia, i.e. intensive proliferation of cells of the tissue, in which the precancerous synkaryon preexist. In the overwhelming majority of cases, because of unsuccessful mitosis (so-called lethal mitosis), elimination, perish of precancerous synkaryons probably takes place at stage of transformation into tumor synkaryons. However in rare cases, in result of specific (reciprocal or nonbalanced) translocations, duplications and following genes' amplifications, these cells may undergo irreversible 
alterations and may transform into true tumor cells (synkaryons of stage II).

From the chromosomal aberrations, the most dangerous in carcinogenic respect are nonbalanced translocations, and also duplications, expressed in "complementation" of chromosomes' identical sites, having the same function. This event usually leads to genes' amplification, in consequence of which expression of genes (oncogenes), responsible for the control under the cellular proliferation, ultimately may originate. After this, so-called "over-expressed gene" arose.

In the process of tumor progression, segregation of some chromosomes in the tumorous synkaryon, and also involvement last cells by means of fusion of considerably amount of other tumorous cells and normal cells of different types and maturity take place. After this tumorous cells with extreme polymorphism of karyotype and new abilities, arise. Notwithstanding the fact that a tumor substrate originates initially from one synkaryon, or despite its clonal character, in most cases, the tumorous cells of higly morphologic and cytogenetic polymorphism originate. Cellular subpopulations are constantly formed in them tumor focus without any obvious regularity and can coexist in the phenotypically and genotypically distinguished cells.

Thus, malignant tumors have clonal origin and on initial stage consist of genetically identical cells. In the future, cellular substrate of tumors develop continuously and alters genetic properties. As it was shown, in most cases, malignant neoplasms in late stages of carcinogenesis (progression) are heterogenic in antigen composition, as by metastatic abilities, by character of growth on plastic substrate, and in cytogenetic, ultrastructure and histochemical signs as well [8-10]. Karyogamic theory of carcinogenesis or the theory of "two synkaryons" explaines these facts by spontaneous or induced somatic hybridization between tumorous cells, or tumorous and precancerous cells, or between tumorous and normal cells of tumor substrate.

Thus, it could be concluded that physical, chemical, and biological carcinogens and influences lead to similar result: to induction of cells malignization by means of somatic hybridization. Consequently, hybridization of somatic cells represents itself as one of the possible mechanism of malignant conversion. Formed as a result of this process heterokaryons, homokaryons and synkaryons are to be considered as basis (substratum) of malignant growth. Conclusion could be made also, that malignant growth is polyetiologic, and at the same time monopathogenetic process.

At the same time, in distinction from the generally accepted modern views in oncology, which consider, that initiated agents influence the cells' genotype (genotropic, genotoxic actions), by the karyogamic theory of carcinogenesis (or the theory of "two synkaryons"), initiated agents in the first instance interact with the cells' plasma membranes, inducing their perforations, fusion process and only then cells' somatic hybridization (i.e. quantitative aberrations of chromosomes) [11-14].

\section{Conclusions}

Here we are trying to answer one of the most complex questions in oncology from the view of karyogamic theory: are the chromosomal aberrations the cause or the consequence of the cancerous growth? We hypothesize that during initiation (formation of precancerous cells) chromosomal aberrations (quantitative aberrations) are on the third place after the perforations of the plasmatic membrane and fusogeny (formation of dikaryons). During promotion (i.e. formation of true cancerous cells) chromosomal aberrations (first of all structural aberrations) are on the first place before the gene amplification. In the case of progression (formation of cancerous cells with a new genotypes and phenotypes) the sequence should be the same as for the initiation (perforations, fusogeny and after that chromosomal aberrations). So the role of chromosomal aberrations differs at the different stages of carcinogenesis, but there are no doubts about their causative connections: at the stages of initiation and progression chromosomal aberrations are among the main causative factors (after the plasmatic membrane perforations and fusogeny), while at the stage of promotion, the main causative factor is the structural chromosomal aberrations [15].

Thus, chromosomal aberrations (as quantitative, as structural) are one of the direct reasons of tumorous process, but not its consequence. These chromosomal aberrations originated only after fusion of somatic cells, in particular, from a moment of precancerous cell arising; i.e., on initial phase take place perforations of cell's plasma membranes by carcinogens agents, then, arising of dikaryons (hetero- and homokaryons), and only an moment of arising of precancerous cell (synkaryon of stage I), begin of genetical processes involving. After above-marked conversion on subcellular and molecular levels there may arise true tumor synkaryon (so-called synkaryon of stage II), malignant cell with the ability of uncontrolled proliferation. Synkaryon of stage II, represents clone, from which formation of malignant tumors substrate on early stage of carcinogenesis begins.

\section{REFERENCES}

[1] Cohen SM, Arnold LL. Chemical carcinogenesis. Toxicological Sciences, 2011, 120 (suppl.1), 576-592.

[2] Zilber LA. Virus-genetic theory of tumor formation. Moscow, 1968.

[3] Minuk GY, Zhang M, Gong Y. et al. Decreased hepatocyte membrane potential differences and BABAa-B3 expression in human hepatocellular carcinoma. Hepatology, 2007, 45, 3 : 735-745.

[4] Vaananen, P; Kaarianen, L. Fusion and haemolysis of erythrocytes caused by three togaviruses; Semliki Forest, Sindbis and Rubella. J Gen Virol 1980; 46: 467-475. 
[5] Huang, RT; Rott, R; Klenk, HD. Influenza viruses cause haemolysis and fusion of cells. Virology 1981; 110: 243-247.

[6] White G., Metlin K., Helenius A. Cell fusion by Semliki Forest, influenza, and vesicular stomatitis viruses. J Cell Biol., 1981, 89: 674-679.

[7] Owens RJ, Burke C, Rose JK. Mutations in the membrane-spanding domain of the human immunodeficiency virus envelope glycoprotein that affect fusion activity. J Virol, 1994, 68: 570-574.

[8] Nowell, PS. The clonal evolution of tumor cell populations. Science 1976; 194: 23-28.

[9] Woodruff, MFA. What's going on in the cancer patient ? Pathology 1986; 18: 175-180

[10] Leder, LD. Zur individualitat von malignen neoplasien. Strahlanther und Oncol 1986; 162: 624-628.
[11] Gogichadze GK., Misabishvili EV., Gogichadze TG. Tumor cells formation by normal somatic cells fusing and cancer prevention prospects. Medical Hypotheses, 2006, 66, 1, 133-136.

[12] Gogichadze GK., Gogichadze TG. Karyogamic theory of cancer cell formation from the view of the XXI century. Nova Biomedical Books, New York, 2010.

[13] Gogichadze GK. Common characteristics of a cancer cell and logical corroborations of its hybrid essence. Advances in Genetic Research. Ed. Kevin Urbano, 2011, 46, 161-178.

[14] Gogichadze GK., Gogichadze TG. Somatic hybridization, as a primary reason of malignization. Lambert Academic Publisher, Saarbruken, 2013.

[15] Wolman, SR. Cytogenetic heterogeneity: its role in tumor evolution. Cancer Gene. Cytogenet 1986; 19: 129-140. 